[Agr. Biol. Chem., Vol. 34, No. 11, p. 1751 1753, 1970]

\title{
Effect of 4-Thiouridine on Chloroplast Development
}

Sir:

The presence of 4-thiouridine $(4 \mathrm{~s} \mathrm{U})$ as one of minor constituents has been established in transfer RNAs from Escherichia coli ${ }^{11}$ and from Mycoplasma." Reports regarding the effects of $4 \mathrm{~s} U$ to organisms, however, have not yet been published, though one of us demonstrated that $4 \mathrm{~s} U$ showed specific photochemical behaviors distinguished from other constituents of RNAs. ${ }^{3 !}$ In this paper we wish to report the inhibitory effect of $4 \mathrm{sU}$ on the chloroplast development in higher plant cotyledons.

Radish seeds (Raphanus sativus Linn. Kaiware) obtained from Takii Shubyo Co., Ltd., Kyoto, were allowed to germinate on $0.5 \mathrm{mM} 4 \mathrm{sU}$ containing $5 \mathrm{mM}$ potassium phosphate buffer $(\mathrm{pH} 7.0)$ in the dark at $22^{\circ} \mathrm{C}$ for 4 days, and etiolated seedlings obtained were illuminated with Mitsubishi fluorescent lamps (4000 lux) at $22^{\circ} \mathrm{C}$ for the desired time. Controls were left under the same conditions except the presence of $4 \mathrm{~s} U$. Germination and growth of the seeds were not affected by the presence of $0.5 \mathrm{~mm} 4 \mathrm{sU}$, the rate of chlorophyll synthesis in the cotyledons on illumination, however, was significantly delayed, compared with that of the untreated plants, as shown in Fig. 1. The result indicates that the contact with $4 \mathrm{~s} U$ in the germination stage should be primarily related to the inhibition of the greening on illumination. Cysteine, glutathione, 6-mercaptopurine, uridine, uracil, thiourea or glucose (l mM, respectively) had no inhibitory effect

1) M. N. Lipsett, J. Biol. Chem., 240, 3975 (1965).

2) H. Hayashi, H. Fisher and D. Söll, Biochemistry, 8, 3680 (1969).

3) M. Pleiss, H. Ochiai and P. A. Cerutti, Biochem. Biophys. Res. Comm., 34, 70 (1969). on the greening. Glutathione and plant hormones such as 6-benzyl adenine, gibberellin $\mathrm{A}_{3}$ and indole acetic acid had no positive restoration effect against the inhibition induced by $4 \mathrm{sU}$. Interestingly germination of the seeds was suppressed by 2-thiouracil $(0.5 \mathrm{mM})$ almost perfectly.

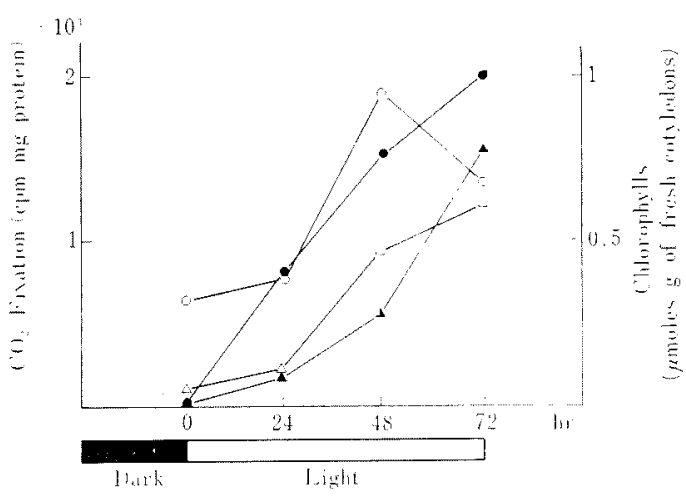

FIG. 1. Comparative Study on Chlorophylls and RuDP Carboxylase in Radish Cotyledons Cultured with and without $4 \mathrm{~s} U$.

Radish seeds were allowed to germinate in the dark for 4 days and etiolated seedlings obtained were illuminated for 3 days. Chlorophyll content and the enzyme carboxylase activity in cotyledons were determined at $0,24,48$ and $72 \mathrm{hr}$ of illumination; chlorophyll was extracted with $80^{\circ}$ acetone and determined according to the method of D. I. Arnon," the enzyme was assayed according to the J. Hurwitz's method ${ }^{5}$ using ${ }^{14} \mathrm{C}-\mathrm{NaHCO}_{3}$.

$\mathrm{O}-\mathrm{O}$, RuDP carboxylase without $4 \mathrm{sU}$;

$\triangle-\triangle$, RuDP carboxylase with $4 \mathrm{~s} \mathrm{U}$;

- Chlorophylls without $4 \mathrm{sU}$;

$\Delta-\Delta$, Chlorophylls with $4 \mathrm{~s} \mathrm{U}$.

4) D. I. Arnon, Plant Physiol., 24, 1 (1949).

5) B. Filner and A. O. Klein, Plant Physiol., 43, 1587 (1968). 
Determination of protochlorophyllide, a precursor of chlorophyll, after the Siegelman's method $^{6}$ indicated that the formation of protochlorophyllide in cotyledons was also prevented by the presence of $4 \mathrm{sU}$. Furthermore phototransformation of protochlorophyllide holochrome, which was isolated from normal etiolated bean cotyledons after the Siegelman's method, ${ }^{61}$ to chlorophyllide holochrome with illumination was not inhibited by the presence of $0.5 \mathrm{mM} 4 \mathrm{sU}$. Delta-aminolevulinic acid, a starting material of chlorophyll biogenesis, was demonstrated to accumulate in $40 \pm 10, \%$ excess in the $4 \mathrm{sU}$ treated cotyledons as compared with controls.

The specific activity of glucose 6-phosphate dehydrogenase (G6PDH, E. G. 1.1.1.49, DGlucose-6-phosphate: NADP oxidoreductase), ${ }^{7}$ a typical enzyme in the soluble cytoplasm, in the cotyledons grown on $4 \mathrm{sU}$, was the same as the activity of controls. Glutamic acid dehydrogenase (GluDH, E. C. 1.4.1.3, LGlutamate: NAD oxidoreductase), ${ }^{81}$ a typical enzyme of mitochondria, also showed no difference in activity between the $4 \mathrm{~s} \mathrm{U}$ treated plants and controls as shown in Fig. 2. However the specific activity of ribulose 1,5-diphosphate(RuDP) carboxylase (E. C. 4.1.1.39, 3-Phospho-D-glycerate carboxy-lyase), ${ }^{5}$ which is a proper enzyme of chloroplast, was low in etiolated cotyledons germinated with $4 \mathrm{sU}$ but significantly high in the cotyledons without $4 \mathrm{~s} U$. By continuous illumination the activity of the enzyme was restored to the normal value after 3 days as well as the chlorophyll content as shown in Fig. 1.

In addition, analysis by the use of polyacrylamide gel electrophoresis of cotyledon

6) P. Schopfer and H. W. Siegelman, Plant Physiol., 43, 990 (1968).

7) A. Kornberg and B. L. Horecker, "Methods in Enzymology," Vol. I, ed. by S. P. Colowick and N. O. Kaplan, Academic Press Inc., New York, N.Y., 1955, p. 323.

8) G. Beisenherz, Th. Bücher et al., Z. Naturforsch, 86, 555 (1953).
$\mathrm{RNAs}^{9,101}$ from normal or $4 \mathrm{sU}$ treated radis demonstrated that the rate of de novo bic synthesis of chloroplast RNAs induced b illumination was significantly retarded in th $4 \mathrm{~s} \mathrm{U}$ treated plants as compared with that 0 the untreated, whereas the formation of ribc somal RNAs was virtually analogous in th both cotyledons.

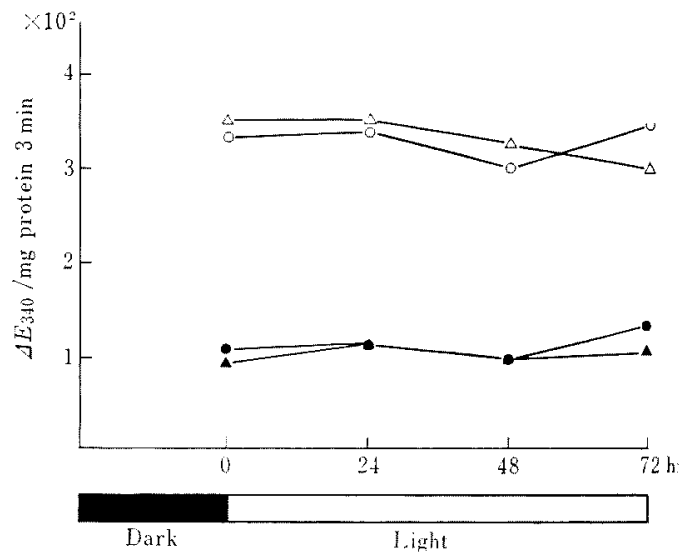

FIG. 2. Comparative Study on G6PDH and GluDH Activities in Radish Cotyledons Cultured with and without $4 s \mathrm{U}$.

G6PDH was assayed according to the A. Kornberg's method,7) GluDH according to the Th. Bücher's. ${ }^{8}$ Specific activity was determined by measurement of $\Delta E_{340} / \mathrm{mg}$ protein for the initial 3 min using $\mathrm{NADP}^{+}$and $\mathrm{NAD}^{+}$as cofactor, respectively.

O-O, G6PDH without $4 \mathrm{sU}$;

$\triangle-\triangle$, G6PDH with $4 s \mathrm{~s}$;

- GluDH without $4 \mathrm{sU}$;

$\Delta-\Lambda$, GluDH with $4 \mathrm{sU}$.

The experimental results described abovi indicate that $4 \mathrm{~s} U$ must have prevented chloro plast development or formation, but no cytoplasmic and mitochondrial development during germination in the dark followed $b$ :

9) J. Ingle, Plant Physiol., 43, 1850 (1968).

10) A.C. Peacock and C.W. Dingman, Biochemistry 7, 668 (1968). 
growth in the light. It is interesting that the

Hideo OCHIAI

effect of $4 \mathrm{sU}$ disapperared by continuous illu-

Hitoshi ShIBata

mination. Details and electron microscopic

studies on these phenomena will be reported elsewhere in the near future.

Laboratory of Biochemistry, Colledge of Agriculture,

Shimane University, Matsue, Shimane, Japan

Received August 31, 1970 